\title{
The kinematics and chemistry of the Sagittarius streams
}

\author{
Weibin Shi, ${ }^{1,2}$ Yuqin Chen, ${ }^{1}$ Kenneth Carrell, ${ }^{1}$ and Gang Zhao ${ }^{1,2}$ \\ ${ }^{1}$ Key Laboratory of Optical Astronomy, National Astronomical Observatories, \\ Chinese Academy of Sciences, Beijing 100012, China \\ email: swb@sdu.edu.cn \\ ${ }^{2}$ Shandong Provincial Key Laboratory of Optical Astronomy and the Solar-Terrestrial \\ Environment, School of Space Science and Physics, Shandong University at Weihai, \\ Weihai 264209, China
}

\begin{abstract}
We selected red horizontal-branch stars along the streams of the Sagittarius dwarf galaxy (Sgr) from SDSS DR7 spectroscopic data using a theoretical model. We investigate the metallicity distribution of stars in the Sgr streams. The metallicity gradient is $(-1.8 \pm 0.3) \times 10^{-3}$ dex $\operatorname{deg}^{-1}$ in the first wrap of the trailing arm and $(-1.5 \pm 0.4) \times 10^{-3} \mathrm{dex} \mathrm{deg}^{-1}$ in the first wrap of the leading arm.
\end{abstract}

Keywords. Galaxy: halo, stars: horizontal-branch

\section{Introduction}

The Sagittarius (Sgr) dwarf galaxy is the second nearest galaxy to our Milky Way (assuming that the Canis Major dwarf galaxy is the nearest). The Sgr galaxy is currently being disrupted under the strain of the Milky Way. Studying the metallicity and kinematic distributions of Sgr stars has now become an important issue. Thus far, metallicity and abundance studies of the Sgr tails have been less detailed if they were based on large samples of stars located in various locations. We analyze the metallicity distribution at different points along the tidal streams of the Sgr dwarf based on low-resolution data for a large sample of stars.

\section{Sample selection}

We obtained red horizontal-branch (RHB) stars from the sDSS DR7 low-resolution spectral data (Chen et al. 2010). We chose the Sgr stars with the aid of the theoretical model of Law \& Majewski (2010). First, we obtained stars from the full RHB stellar sample using R.A. and Dec positions. Second, we chose RHB stars in the Sagittarius leading and trailing tidal tails using a Distance $-\Lambda_{\odot}$ map, where $\Lambda_{\odot}$ is the Sgr longitude scale along the orbital plane. Third, we selected likely member stars of the Sgr stream based on their radial velocities, which should be appropriate for the Sgr stream at these positions, based on Sgr debris models (Chou et al. 2010).

\section{The metallicity gradient along the leading and trailing arms}

There is a metallicity gradient along trailing arm 1, while there is a lower-significance gradient along leading arm 1 . We find that in the trailing arm, when moving farther from the Sgr core along arm 1 and onward to arm 2, the typical metallicity shifts to more metal-poor values. This suggests an evolution toward more ancient stars, since 


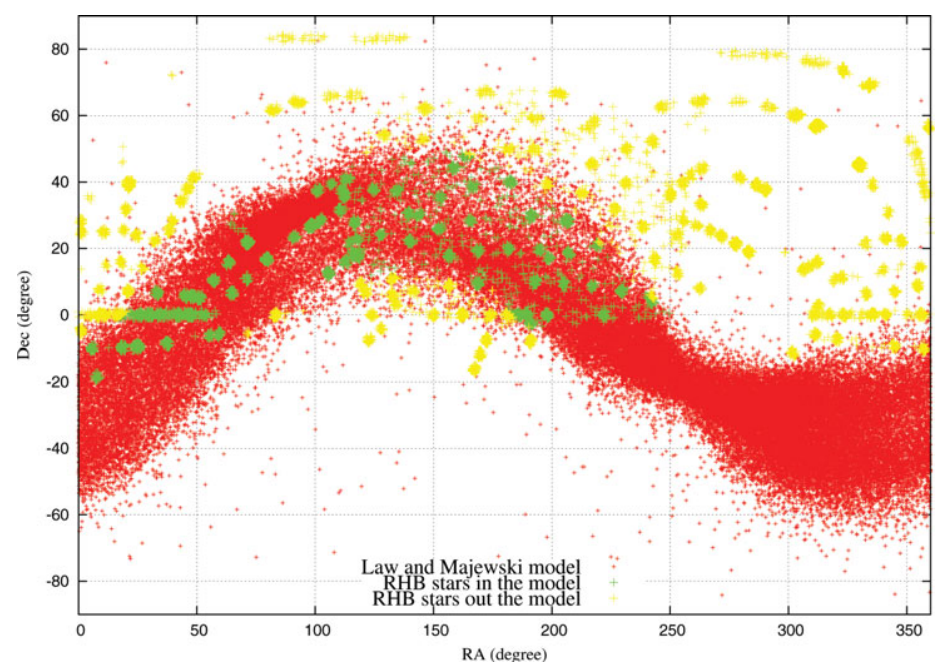

Figure 1. SDSS RHB stars based on the Law \& Majewski (2010) model (red points) in an R.A-Dec map. We selected 3512 stars (green points) from a total of 8535 RHB stars.

\section{Leading arm 1}

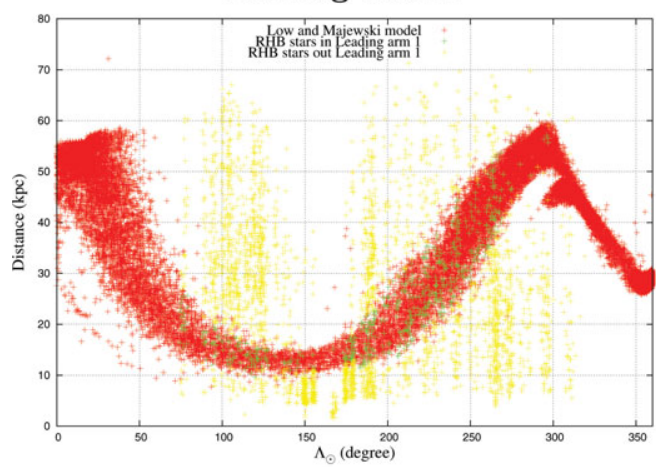

Trailing arm 1

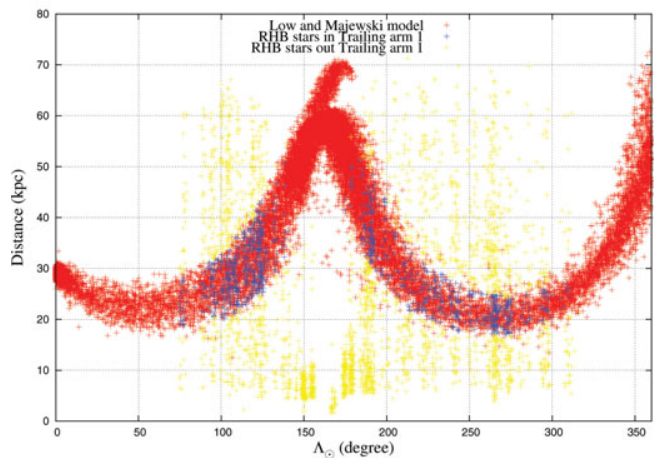

\section{Leading arm 2}

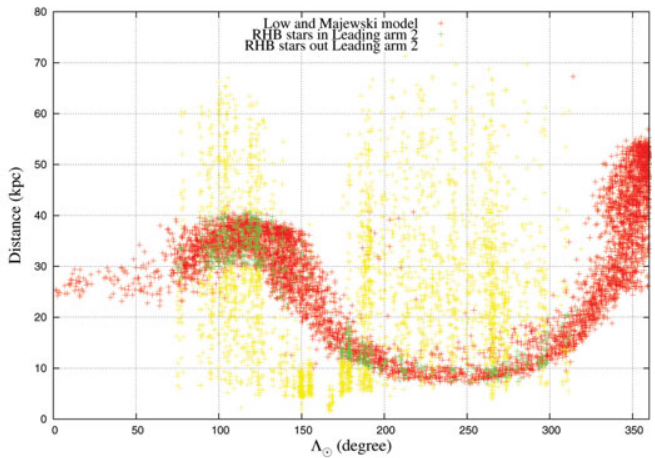

Trailing arm 2

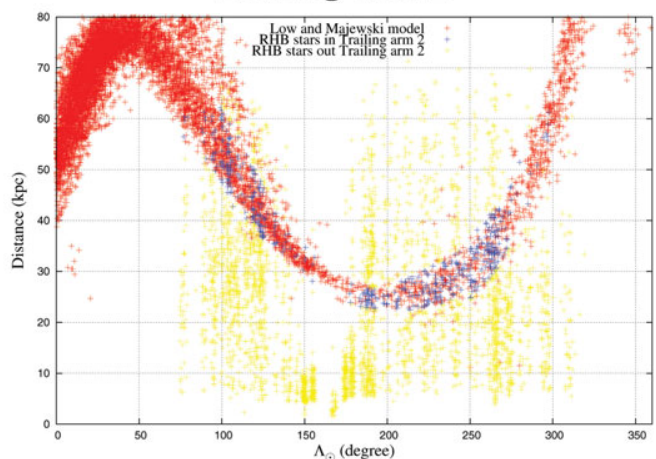

Figure 2. Selected RHB stars based on the Law \& Majewski (2010) model (red points) in a Distance- $\Lambda_{\odot}$ map. We obtained 586 stars (green points) in Leading arm 1 and 585 stars (green points) in Leading arm 2 from our 3512 sample stars (green and yellow points). We obtained 973 stars (blue points) in Trailing arm 1 and 502 stars (blue points) in Trailing arm 2 from the 3512 stars in our sample (blue and yellow points). The first (second) wrap of the Law \& Majewski (2010) model is denoted by the number ' 1 ' ('2'). 
metal-poor RHB stars must be older than metal-rich RHB stars. This is in agreement with dwarf galaxy formation theories, where the more metal-rich core of the galaxy is surrounded by older and more metal-poor stars, since it is this outer, older and more metal-poor population that will be tidally stripped before the younger, inner component.

\section{Conclusions}

In this paper we present the properties of the metallicity distribution for a large sample of RHB stars belonging to the Sgr tidal streams. There are metallicity gradients along the streams of Sgr, $(-1.8 \pm 0.3) \times 10^{-3} \mathrm{dex} \mathrm{deg}^{-1}$ in trailing arm 1 and $(-1.5 \pm 0.4) \times 10^{-3}$ dex $\mathrm{deg}^{-1}$ in leading arm 1 . No significant gradient is present along either trailing arm 2 or leading arm 2. Stars belonging to more ancient wraps of the streams in arm 2 are more metal-poor. Anticipating the upcoming LAMOST spectroscopic survey (Zhao et al. 2006), we can expect to extend our analysis of RHB stars in the Sgr dwarf galaxy to an even larger sample.

\section{Acknowledgements}

This work was supported by the National Natural Science Foundation of China (grants 11178013, 11073026, 11150110135, 11233004, 10933001, and 11273026), and by the Provincial Natural Science Foundation of ShanDong (grant ZR2010AM006).

\section{References}

Chen, Y. Q., Zhao, G., Zhao, J. Z., et al. 2010, AJ, 140, 500

Chou, M., Cunha, K., Majewski, S. R., et al. 2010, ApJ, 708, 1290

Law, D. \& Majewski, S. 2010, ApJ, 714, 229

Zhao, G., Chen, Y. Q., Shi, J. R., et al. 2006, ChJAA, 6, 265 